\title{
Seismic barriers: theory and numerical simulations
}

\author{
Vladimir Bratov ${ }^{1,3^{*}}$, Alla Ilyashenko ${ }^{2},{\text { Nikita } \text { Morozov }^{3} \text { and Tursunbai Rashidov }}^{4}$ \\ ${ }^{1}$ St Petersburg State University, Universitetskaya Emb, 13B, St Petersburg 199034, Russia \\ ${ }^{2}$ Moscow State University of Civil Engineering, Yaroslavskoye Shosse, 26, Moscow, 129337, Russia \\ ${ }^{3}$ Institute for Problems in Mechanical Engineering RAS, Bolshoj pr. 61, St Petersburg, 199178, \\ Russia \\ ${ }^{4}$ Institute of Mechanics and Seismic Stability of Structures by N.M.Urazbaev, Do'rmon yo'li" St 31, \\ Tashkent, 100125, Uzbekistan
}

\begin{abstract}
The basic idea of a seismic barrier is to protect an area occupied by a building or a group of buildings from seismic waves. Depending on the nature of seismic waves that are the most probable in a specific region, different kinds of seismic barriers are suggested. For example, vertical barriers resembling a wall in a soil can protect from Rayleigh and bulk waves. The FEM simulation reveals that to be effective, such a barrier should be (i) composed of layers with contrast physical properties allowing "trapping" of the wave energy inside some of the layers, and (ii) depth of the barrier should be comparable or greater than the considered seismic wavelength.
\end{abstract}

\section{Introduction}

\subsection{Methods of seismic protection}

Generally, current approaches for preventing failure of structures due to seismic activity can be divided into two groups: (i) approaches for creating seismically stable structures and joints; this group contains different methods ensuring either active or passive protection; and (ii) approaches for creating a kind of seismic barrier preventing seismic waves from transmitting wave energy into a protected region.

\footnotetext{
*Corresponding author: vladimir@bratov.com
} 
While the first group includes a lot of different engineering approaches and solutions, the second one contains very few studies; see Takahashi et al. (2001) and more recent works by Motamed et al. (2008), Kusakabe et al. (2008). The proposed research belongs to the second group.

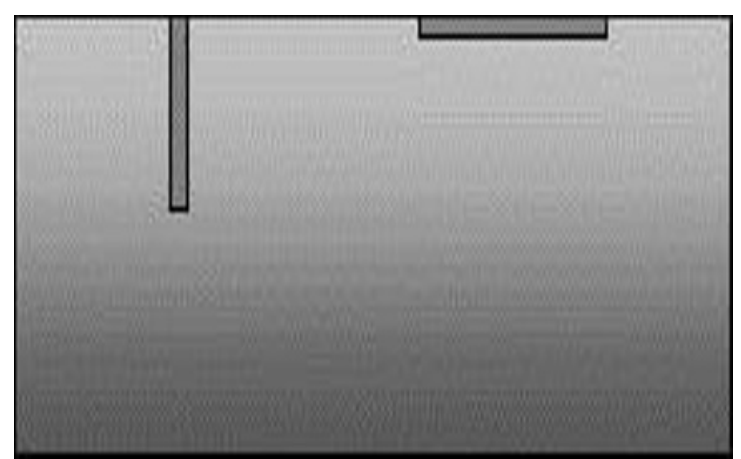

Fig. 1. Vertical and horizontal seismic barriers.

\subsection{Possible types of wave barriers}

The considered seismic barriers can be of two types: vertical, aimed to reflect, trap, and dissipate most of the seismic wave energy; and horizontal, based on Chadwick and Smith (1977) and Love (1911) theorems, and aimed to prevent certain types of seismic waves from propagation; see, Fig. 1.

Yet another interesting approach is to create a "rough" surface of the half-space to force the propagating Rayleigh wave scatter by caves and swellings; see Fig.2, where part of a free surface with the sinusoidal roughness is pictured. In this respect, the rough surface apparently transforms the elastic half-space into viscoelastic one. To be effective, periodic imperfections should have magnitude and period comparable to the magnitude and

\section{Rough surfice}

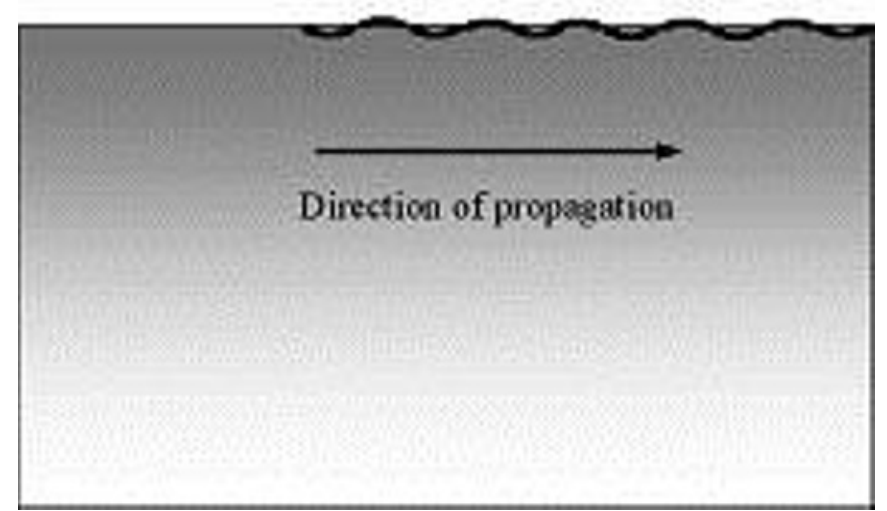

Fig. 2. Rough surface acting as seismic barrier against Rayleigh waves. 
wavelength of propagating Rayleigh wave (Sobczyk 1966, Maradudin \& Mills 1976, Maradudin \& Shen 1980).

In practice, such a rough surface can be achieved by a series of rather deep trenches oriented transversally to the most probable direction of the wave front. Some of obvious deficiencies of this method are: (i) its inability to persist the surface waves other than Rayleigh waves; (ii) protection from Rayleigh waves travelling only in directions that are almost orthogonal to orientation of the trenches; and, (iii) high sensitivity to the frequency of travelling Rayleigh waves. These shortcomings made an idea of exploiting a rough surface as a kind of protective barrier, unrealizable.

\subsection{Vertical barriers}

For bulk waves the most effective vertical barrier would be an empty trench, or a trench filled in with a lighter material than the ambient soil. For such a barrier most of the wave

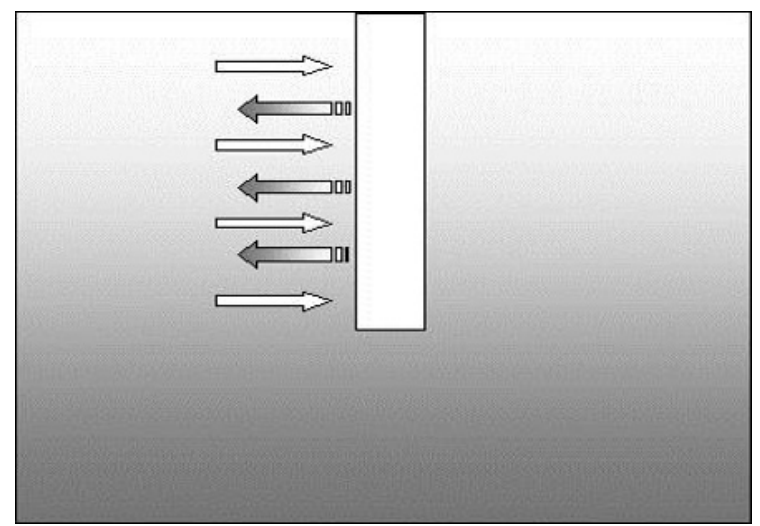

Fig. 3. Full reflection of an incident bulk wave from an empty trench.

energy would be reflected, as is shown on Fig.3.

However, propagating Rayleigh or Love wave will simply overflow an empty trench, as Fig.4 shows.

Thus, to be effective against the most dangerous types of seismic Rayleigh and Love waves, the vertical barrier should be of a more elaborate type. Possible structures of vertical barriers will be discussed later on.

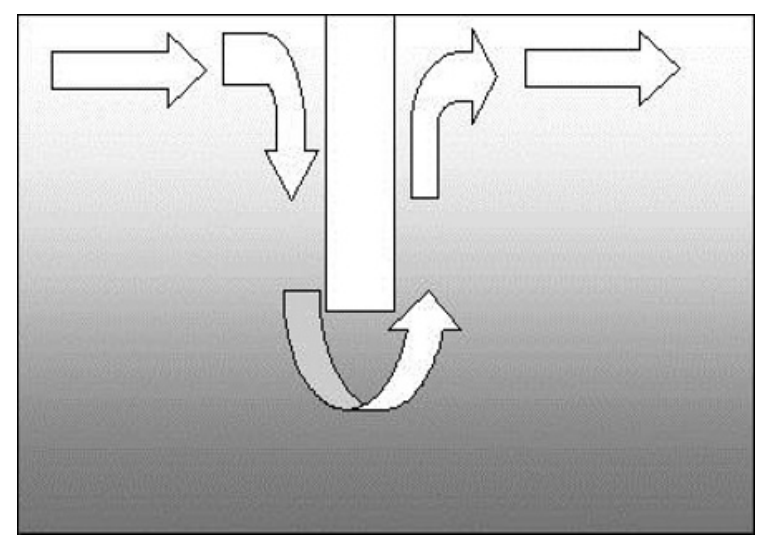

Fig. 4. Flow of Rayleigh wave around an empty trench 


\subsection{Horizontal barriers}

Horizontal barriers can be constructed by modifying properties of the outer layer preventing the corresponding surface wave from propagation.

In practice, modifying physical properties of the outer layer can be achieved by reinforcing ground with piles or "soil nails"; see papers where reinforcing was studied for increasing bearing load of the soil (Blondeau 1989, De Buhan et al. 1989, Abu-Hejleh et al. 2002, Eiksund 2004, Herle 2006).

If distance between piles is sufficiently smaller than the wave length, then a reinforced region can be considered as macroscopically homogeneous and either transversely isotropic or orthotropic depending on arrangement of piles. Of course, homogenized physical properties of the reinforced medium depend upon material of piles, distance between them, and their arrangements.

\section{Seismic surface acoustic waves}

In this section we proceed to analyzes of the main types of seismic surface waves and conditions for their non-existence

\subsection{Rayleigh waves}

These waves discovered by Lord Rayleigh (Strutt 1885) propagate on a plane surface of a halfspace; and exponentially attenuate with depth. These waves transmit the most seismic energy and lead to most severe damage in earthquakes.

One interesting problem associated with Rayleigh waves is a problem of "forbidden" directions of "forbidden" (necessary anisotropic) materials that does not transmit a Rayleigh wave along some directions. Forbidden materials and forbidden directions have been intensively searched both experimentally and numerically (Lim \& Farnell 1968, 1969, Farnell 1970) until mid seventies when the theorem of existence for Rayleigh waves was rigorously proved (Barnett \& Lothe 1973, 1974a,b, Lothe \& Barnett 1976 Chadwick \& Smith 1977, Chadwick \& Jarvis 1979, Chadwick \& Ting 1987). This theorem states that no materials possessing forbidden directions for Rayleigh waves can exist.

Despite proof of the theorem of existence, a small chance for existence of forbidden materials remained. This corresponded to the case of non-semisimple degeneracy of a special matrix associated with the first-order equation of motion; actually, this matrix is the Jacobian for the Hamiltonian formalism used for Rayleigh wave description. However, it was shown (Kuznetsov 2003) that even at the non-semisimple degeneracy a wave resembling the genuine Rayleigh wave can propagate. Thus, for waves propagating on a homogeneous half-space, no forbidden materials or directions can exist.

\subsection{Stoneley waves}

These are waves were introduced by Stoneley (1924), and analyzed by (Sezawa \& Kanai 1939, Cagniard 1939, Scholte 1947). Stoneley waves propagate on an interface between two contacting half-spaces.

In contrast to Rayleigh waves, Stoneley waves can propagate only if material constants of the contacting half-spaces satisfy special (very restrictive) conditions of existence. These conditions were studied by Chadwick \& Borejko (1994), Sengupta \& Nath (2001).

It should be noted that for the arbitrary anisotropy no closed analytical relations between material constants of the contacting half-spaces ensuring existence or nonexistence of Stoneley waves have been found (2010). 


\subsection{Love and SH waves}

Love waves (Love, 1911) are horizontally polarized shear waves that propagate on the interface between an elastic layer contacting with elastic half-space. At the outer surface of the layer traction-free boundary conditions are generally considered.

In the case of both isotropic layer and half-space the conditions of existence derived by Love are:

$$
c_{\text {layer }}^{S}<c_{\text {halfspace }}^{S}
$$

where $c_{*}^{S}$ are the corresponding speeds of the transverse bulk waves. At violating condition (1) no Love wave can propagate. For the case of both anisotropic (monoclinic) layer and a half-space the condition of existence is also known (Kuznetsov 2006a).

$\mathrm{SH}$ waves resemble Love waves in polarization, but differ in absence of the contacting half-space. At the outer surfaces of the layered plate different boundary conditions can be formulated (Kuznetsov 2006b). In contrast to genuine Love waves, the SH waves exist at any combination of elastic properties of the contacting layers.

Besides Love and SH waves a combination of them can also be considered. This corresponds to a horizontally polarized wave propagating in a layered system consisting of multiple layers contacting with a half-space. Analysis of conditions of propagation for such a system can be done by applying either transfer matrix method (Thomson 1950, Haskell 1953), known also as the Thomson-Haskell method due to its originators; or the global matrix method mainly developed by Knopoff (1964).

At present (2010) no closed analytical conditions of existence for the combined Love and $\mathrm{SH}$ waves propagating in anisotropic multilayered systems are known; however, these conditions can be obtained numerically by applying transfer or global matrix methods; see (Kuznetsov 2006a, b; Djeran-Maigre \& Kuznetsov 2008).

Different observations show that genuine Love and the combined Love-SH waves along with Rayleigh and Rayleigh-Lamb waves play the most important role in transforming seismic energy in earthquakes (e.g. Agnew 2002, Braitenberg \& Zadro 2007). But, as we have seen, there is a relatively simple (at least from a theoretical point of view) method for stopping Love and the combined Love and SH waves by modifying the outer layer in such a way that conditions of existence (1) are violated.

\subsection{Lamb and Rayleigh waves}

Lamb waves (Lamb, 1917) are dispersive waves propagating in a homogeneous plate and (if a plate is isotropic) polarized in the saggital plane, similarly to polarization of the genuine Rayleigh waves. It is known (Lin \& Keer 1992, Ting 1996) that Lamb waves can propagate at any anisotropy of the layer and at traction-free, clamped, or mixed boundary conditions imposed on the outer surfaces of the plate. The same result can be extrapolated to a layered plate containing multiple anisotropic homogeneous layers in a contact (Ting 2002). Thus, for Lamb waves no forbidden materials exist.

More interesting from seismological point of view are Rayleigh-Lamb waves. These are dispersive waves propagating in a layered plate contacting with a (homogeneous) halfspace. Rayleigh-Lamb waves in isotropic media are polarized in the saggital plane defined by vectors $\mathbf{V}$ (normal to a median plane) and $\mathbf{n}$ (direction of propagation), as Lamb and Rayleigh waves. Needless to say that Rayleigh-Lamb waves are much more difficult for theoretical studies than Rayleigh or Lamb waves. 


\section{Seismic barriers}

Herein, we present some results on numerical simulation of propagating seismic waves and their interaction with seismic barriers. The presented results were obtained by the explicit FE code implemented on a metacluster computer system.

\subsection{Vertical barriers}

Theoretical analysis and numerical simulations reveal that to effectively protect from Rayleigh and Rayleigh-Lamb waves a vertical barrier (Fig.1) should satisfy several conditions: (i) the barrier should have a composite layered structure composed of vertical layers with contrast physical properties; (ii) depth of the barrier should be comparable to the wavelength of the most probable seismic wave; (iii) the protected zone should be completely surrounded by a barrier to avoid flowing of the seismic wave inside the protected zone.

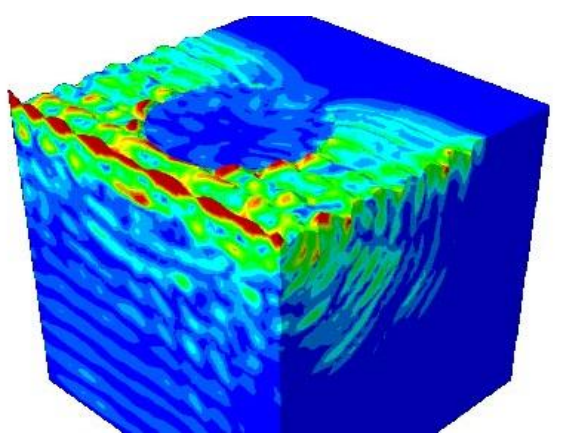

a)

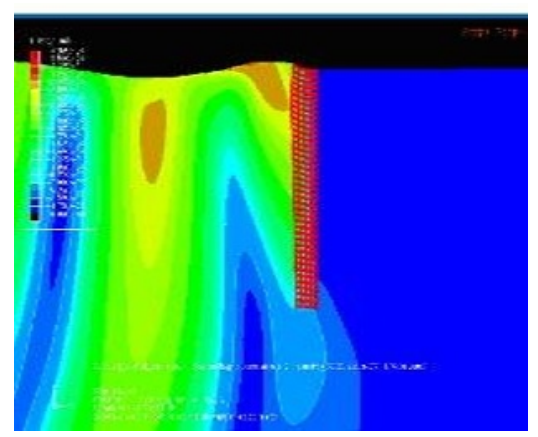

b)

Fig. 5. Round-shaped vertical barrier protecting from Rayleigh waves:

a) $3 \mathrm{D}$ model; b) cross-section near the barrier.

Figure 5 demonstrates a movie frame related to numerical simulation of a propagating seismic Rayleigh wave interacting with a round-shaped vertical barrier; the latter completely surrounds the protected region. The ratio of the wavelength to depth of the barrier was taken $\sim 0.8$. This corresponded to the reference frequency $10 \mathrm{~Hz}$ and the Rayleigh wavelength $20 \mathrm{~m}$ (speed of Rayleigh wave was $200 \mathrm{~m} / \mathrm{sec}$; speed of the transverse bulk wave was $\sim 220 \mathrm{~m} / \mathrm{sec}$ ); diameter of the protected region was $120 \mathrm{~m}$. Inside the protected region reduction of the magnitude of displacements was more than ten times comparing to the outside territory.

\subsection{Transverse (horizontal) barrier}

Our analyses revealed that ssimilarly to vertical barriers, the transverse barriers should satisfy several conditions to effectively protect from seismic waves: (i) length (horizontal) of the barrier should be comparable to the wavelength; (ii) material of the barrier should have larger density than the ambient soil for Rayleigh waves; that is in agreement with Chadwick's theorem stating that at the clamped surface of a halfspace, no Rayleigh wave can propagate; (iii) material of the barrier should satisfy the opposite Love's propagating condition (4) for protecting from propagating seismic Love waves.

Considering propagation of Rayleigh waves or other types of seismic surface waves in soils subjected to liquefaction, Biot's theory of poroelasticity can be applied; see Detournay E. \& Cheng (1993). According to the genuine Biot's theory, all governing equations are linear, that ensures validity of the harmonic wave approach. 
The authors thank the Russian Foundation for Basic Research, Grant 18-58-41001 and (AVI) RFBR Grant 17-08-00311 for partial financial support.

\section{References}

1. Abu-Hejleh, N., Zornberg, J.G., Wang, T. \& Watcharamonthein, J. 2002. Monitored displacements of unique geosynthetic-reinforced bridge abutments. Geosynthetics International. 9(1).

2. Agnew, D.C. 2002. History of seismology. International Handbook of Earthquake and Engineering Seismology. 81(A). 3-11.

3. Balandin, D.V., Bolotnik, N.N., and Pilkey, W.D. 2000. Optimal protection from impact and shock: Theory and methods. Appl. Mech. Rev., ASME. 53(9). 237-264.

4. Balandin, D.V., et al. 2008. Concept of a platform based impact isolation system. Medical Eng. \& Physics. 30(2). 258-267.

5. Barnett, D.M. \& Lothe, J. 1973. Synthesis of the sextic and the integral formalism for dislocations, Green's functions, and surface waves in anisotropic elastic solids. Phys. Norv. $7.13-19$.

6. Barnett, D.M. \& Lothe, J. 1974. Consideration of the existence of surface wave (Rayleigh wave) solutions in anisotropic elastic crystals. J. Phys. Ser. F. $4.671-678$.

7. Barnett, D.M., Lothe, J. 1974. An image force theorem for dislocations in anisotropic bicrystals. J. Phys. Ser. F. 4. $1618-1635$.

8. Bensoussan, A., Lions, J.L., \& Papanicolaou, G. 1978. Asymptotic analysis for periodic structures, North-Holland Publ., Amsterdam.

9. Blondeau, F., 1989. Fondations profondes - Cours de mécanique des sols de l'E.N.P.C. Techniques de l'Ingérieur, Chapitre C, 248.

10. Braitenberg, C. \& Zadro, M. 2007. Comparative analysis of the free oscillations generated by the Sumatra-Andaman Islands 2004 and the Chile 1960 Earthquakes, Bull. Seism. Soc. Amer. 97(1A). S6-S17.

11. Cagniard, L. 1939. Reflexion et refraction des ondes seismique progressive (Thesis), Gauthier-Villars \& Cie., Paris.

12. Cecchi, A., and Rizzi, N.L. 2001. Heterogeneous elastic solids: a mixed homogenization-rigidification technique. Int. J. Solids Struct. 38. 29-36.

13. Chadwick, P. \& Borejko, P. 1994. Existence and uniqueness of Stoneley waves, Geophys. J. Int. 118. 279-284.

14. Chadwick, P. \& Jarvis, D.A. 1979. Surface waves in a prestressed elastic body, Proc. Roy. Soc. London. Ser. A. 366. $517-536$.

15. Chadwick, P. \& Smith, G.D. 1977. Foundations of the theory of surface waves in anisotropic elastic materials. Adv. Appl. Mech. 17. 303 - 376.

16. Chadwick, P. \& Ting, T.C.T. 1987. On the structure and invariance of the BarnettLothe tensors, Quart. Appl. Math. 45. $419-427$.

17. Chimenti, D.E. 1994. Lamb waves in microstructured plates. Ultrasonics. 32. 255260.

18. De Buhan, P., Mangiavacchi, R., Nova, R., Pellegrini, G. \& Salençon, J. 1989. Yield design of reinforced earth walls by a homogenization method. Géotechnique. 39(2). 189-201.

19. Den Hartog, J.P. 1985. Mechanical Vibrations. N.Y., Dover Publ.

20. Detournay E. \& Cheng A. H.-D. 1993. Fundamentals of Poroelasticity, Vol. II Comprehensive Rock Engineering: Principles, Practice \& Projects. N.Y., Pergamon Press

21. Djeran-Maigre, I. \& Kuznetsov, S.V. 2008. Solitary SH waves in two layered traction free plates. Comptes Rendus Acad Sci, Paris, Ser. Mecanique. 336 102-107. 
22. Eiksund, G., Hoff, I. \& Perkins, S. 2004. Cyclic triaxial tests on reinforced base course material. Proceedings EuroGeo3, DGGT, Munich, Germany, Vol. 2, 619-624.

23. Farnell, G.W. 1970. Properties of elastic surface waves. Phys. Acoust. 6. $109-166$.

24. Guo, N. \& Cawley, P. 1993. Lamb wave propagation in composite laminates and its relationship with acousto-ultrasonics, NDT \& E Int., 26, pp. 75-84.

25. Gunderson, S.A., Barnett, D.M., \& Lothe, J. 1987. Rayleigh wave existence theory: a supplementary remark, Wave Motion, 9, 319-321.

26. Haskell, N.A. 1953. Dispersion of surface waves on multilayered media, Bull. Seismol. Soc. America. V.43. No..1. P.17 - 34.

27. Herle, V. 2006. Long-term performance of reinforced soil structures. Proceedings of the 13. Danube-Conference on Geotechnical Engineering, Slovenian Geotechnical Society, Ljublana, Slovenia, Vol. 2, 251-256.

28. Ketchart, K. \& Wu, J.T.H. 2001. Performance test for geosynthetic reinforced soil including effects of preloading. Federal Highway Administration, McLean, VA, USA, Report No. FHWA-R-01-018.

29. Knopoff, L. 1964. A matrix method for elastic wave problems, Bull. Seismol. Soc. America. V.54. No.1. P.431 - 438.

30. Kusakabe O., Takemura J., Takahashi A., Izawa J., \& Shibayama S. 2008. Physical modeling of seismic responses of underground structures, Proceedings of the 12th International Conference of International Association for Computer Methods and Advances in Geomechanics, Goa, India, 1459-1474.

31. Kuznetsov, S.V. 2002. Subsonic Lamb waves in anisotropic plates, Quart. Appl. Math. $60577-587$.

32. Kuznetsov, S.V. 2003. Surface waves of non-Rayleigh type, Quart. Appl. Math. V.61. No.3. P.575 - 582.

33. Kuznetsov, S.V. 2006a. Love waves in monoclinic media (in Russian), Prikl. Math. Mech., vol.70, 141-154.

34. Kuznetsov, S.V. 2006b. SH-waves in laminated plates, Quart. Appl. Math., vol.64, $153-165$.

35. Lamb, H. 1917. On waves in an elastic plate, Proc. Roy. Soc., A93, $114-128$.

36. Lim, T.C. \& Farnell, G.W. 1968. Search for forbidden directions of elastic surfacewave propagation in anisotropic crystals, J. Appl. Phys., 39, 4319- 4325.

37. Lim, T.C. \& Farnell, G.W. 1969. Character of pseudo surface waves on anisotropic crystals, J. Acoust. Soc. Amer., 45, 845 - 851.

38. Lin, W. \& Keer, L.M. 1992. A study of Lamb waves in anisotropic plates, J. Acoust. Soc. Amer., 92, pp. 888-894.

39. Liu, G.R., Tani, J., Watanabe, K., \& Ohyoshi, T. 1990. Lamb wave propagation in anisotropic laminates, J. Appl. Mech., 57, pp. 923 - 929.

40. Lothe, J. \& Barnett, D.M. 1976. On the existence of surface wave solutions for anisotropic elastic half-spaces with free surface, J. Appl. Phys., 47, 428 - 433.

41. Love, A.E.H. 1911. Some Problems of Geodynamics. Cambridge University Press, Cambridge pp. 165-178.

42. Lowe, M.J.S. 1995. Matrix techniques for modeling ultrasonic waves in multilayered media, IEEE Trans. Ultrasonics, Ferroelectrics, and Frequency Control, 42, 525-542.

43. Maradudin A. and Mills D.L. 1975. Scattering and absorption of electromagnetic radiation by a semi-infinite medium in the Presence of surface roughness. Phys. Rev. 11(B) 1392.

44. Maradudin A. and Mills D.L. 1976. Attenuation of Rayleigh surface waves by surface roughness. Appl Phys. Letters. 28(10) 573-575. 
45. Maradudin A. and Shen J. 1980. Multiple scattering of waves from random rough surfaces. Phys. Rev. 22(B) 4234-4240.

46. Michel, J.C., Moulinec, H., \& Suquet, P. 1999. Effective properties of composite materials with periodic microstructure: A computational approach. Comput. Methods Appl. Mech. Eng., 172, 109-143.

47. Moghaddas-Nejad, F. \& Small, J.C. 2003. Resilient and permanent characteristics of reinforced granular materials by repeated load triaxial tests. Geotechnical Testing Journal, ASTM, Vol. 26, Issue 2.

48. Motamed R., Itoh K., Hirose S., Takahashi A., \& Kusakabe O. 2008. Evaluation of Wave Barriers on Ground Vibration Reduction through Numerical Modeling in ABAQUS, Proceedings of SIMULIA Customer Conference 2009, London, UK, 402-41.

49. Sanchez-Palencia, E. 1983. Homogenization method for the study of composite media, Asymptotic Analysis II, 192-214.

50. Scholte, J.G. 1947. The range of existence of Rayleigh and Stoneley waves, Monthly Notices Roy. Astron. Soc.: Geogphys. Suppl., vol.5, pp. 120-126.

51. Sengupta, P.R. \& Nath, S. 2001. Surface waves in fiber-reinforced anisotropic elastic media, Sadhana, 26, $363-370$.

52. Sezawa, K. 1927. Dispersion of elastic waves propagated on the surface of stratified bodies and on curved surfaces. Bull. Earthquake Res. Inst. Tokyo, 3,1 - 18.

53. Sezawa, K. \& Kanai, K. 1939. The range of possible existence of Stoneley waves and some related problems, Bull. Earthquake Research Inst. (Tokyo), vol.17, pp.1-8.

54. Sobczyk K. 1966. Scattering of Reyleigh waves at a random boundary of an elastic body, Proc. Vibr. Problems, 7(4), 363-374.

55. Stoneley, R. 1924. Elastic waves at the surface of separation of two solids, Proceedings of the Royal Society (London) A106, pp. 416-428.

56. Strutt J.W. (Lord Rayleigh) 1885. On wave propagating along the plane surface of an elastic solid, Proc. London Math. Soc. Vol. 17, 4-11.

57. Takahashi A., Takemura J., \& Shimodaira T. 2001. Seismic performance of reinforced earth wall with geogrid, Proceedings of the 15th International Conference on Soil Mechanics and Geotechnical Engineering, Istanbul, Turkey, 1265-1268.

58. Thomson, W.T. 1950. Transmission of elastic waves through a stratified solid medium, J. Appl. Phys. V.21. No.2. P.89-93.

59. Ting, T.C.T. 1996. Anisotropic elasticity: theory and Applications, Oxford University Press.

60. Ting, T.C.T. 2002. An explicit secular equation for surface waves in an elastic material of general anisotropy, Q. J. Mech. Appl. Math, 55, 297-311. 